\title{
Power Spectrum of Cosmic String Perturbations on the Microwave Background
}

\author{
Invited Talk at the conference 'Unified Symmetry of the Small and the \\ Large', January 27-30 1994, Miami, Florida. \\ Leandros Perivolaropoulos円 \\ Division of Theoretical Astrophysics \\ Harvard-Smithsonian Center for Astrophysics \\ 60 Garden St. \\ Cambridge, Mass. 02138, USA.
}

\begin{abstract}
I review recent progress towards a detailed understanding of the power spectrum of cosmic string perturbations induced on the Microwave Background (CMB). The use of a simple analytic model allows to include important effects that have not been included in previous studies. These effects are potential fluctuations on the last scattering surface (the Sachs-Wolfe effect) and Doppler CMB perturbations. These previously neglected fluctuations are shown to dominate over post-recombination fluctuations (Gott-Kaiser-Stebbins effect) on angular scales less than about $2^{\circ}$ assuming that no reionization occurs. The effective power spectrum index $n_{\text {eff }}$ is calculated on COBE scales and is shown to be somewhat larger than $1\left(1.35 \lesssim n_{\text {eff }} \lesssim 1.5\right)$. After normalizing the model on COBE data and string simulations, I derive the predicted values of $\left(\frac{\delta T}{T}\right)_{r m s}$ for several ongoing CMB experiments on medium and small angular scales. A comparison is also made with current observations and predictions of models based on inflation.
\end{abstract}

\footnotetext{
${ }^{1}$ E-mail address: leandros@cfata3.harvard.edu
} 


\section{Introduction}

One of the most important characteristics of a model for large scale structure formation is the spectrum of primordial perturbations the model predicts. This spectrum is imprinted on the last scattering surface and parts of it show up on the cosmic microwave background (CMB) temperature maps of experiments at various angular scales.

The CMB power spectrum predicted by models based on inflation 22, 3] has been well studied analytically [4] on all scales larger than 1-2 arcmin. The main competition to these models comes from models where the primordial perturbations are generated by topological defects which produce seed-like primordial perturbations. These perturbations are imprinted on the CMB as temperature perturbations and can be detected by ongoing CMB experiments. A particularly interesting type of topological defect is the cosmic string.

The cosmic string theory [5, 6, 7] for structure formation is the oldest and (together with textures [8]) best studied theory of the topological defect class. By fixing its single free parameter $G \mu$ ( $\mu$ is the effective mass per unit length of the wiggly string and $G$ is Newtons constant) to a value consistent 9] with microphysical requirements coming from GUT's, the theory may automatically account for large scale filaments and sheets 10, 11, 12, 13, 14, 15, galaxy formation at epochs $z \sim 2-3$ 116, 17 and galactic magnetic fields [18]. It can also provide large scale peculiar velocities [19, 20] and is consistent with the amplitude, spectral index [21, 22, 23, 24, 25, 26] and the statistics 27, 28, 29, 30] of the cosmic microwave background (CMB) anisotropies measured by the COBE collaboration [31, 32] on large angular scales $\left(\theta \sim 10^{\circ}\right)$.

The CMB spectrum of the cosmic string model has been investigated using both simulations 22, 24 and analytical methods 25 but only on large angular scales (i.e. scales of a couple of degrees or larger). This paper shows the results of an attempt I have recently made 26 to extend the analysis of the CMB spectrum predicted by cosmic strings to arbitrarily small scales. Due to resolution limitations this extension can only be made using analytical methods.

In the next section I will briefly review the three basic mechanisms by which strings can induce perturbations on the CMB: post-recombination Gott-Kaiser-Stebbins [33, 34] (GKS) type perturbations, potential perturba- 
tions on the last scattering surface (LSS) and Doppler perturbations due to velocities of electron last scatterers[35]. Then I will describe an analytical method [19, 25, 28, 36, 20] used to derive the power spectrum of CMB fluctuations induced by strings, in terms of the single free parameter of the model $(G \mu)$ and three evolution parameters that can be fixed from string simulations. In section 3 , I will show how can these parameters be fixed by using the COBE detection for the free parameter $G \mu$ and string simulations for the evolution parameters. The scale invariance of the spectrum will also be demonstrated. Finally, in section 4, I will use the resulting normalized spectrum to make predictions for the rms temperature fluctuations detected by ongoing CMB experiments on medium and small angular scales.

I will assume $\Omega_{0}=1 h=1 / 2$, Cold Dark Matter (CDM), $\Lambda=0$ and standard recombination.

\section{Types of CMB Fluctuations}

The best studied mechanism for producing temperature fluctuations on the CMB by cosmic strings is the Gott-Kaiser-Stebbins (GKS) effect 33, 34]. According to this effect, moving long strings present between the time of recombination $t_{r e c}$ and today produce (due to their deficit angle[37]) discontinuities in the CMB temperature between photons reaching the observer through opposite sides of the string.

The second mechanism for producing CMB fluctuations by cosmic strings is based on potential fluctuations on the LSS. Long strings and loops present between the time of equal matter and radiation $t_{e q}$ and the time of recombination $t_{r e c}$ induce density and velocity fluctuations to their surrounding matter. These fluctuations grow gravitationally and at $t_{r e c}$ they produce potential fluctuations on the LSS. Temperature fluctuations arise because photons have to climb out of a potential with spatially dependent depth.

The third mechanism for the production of temperature fluctuations is based on the Doppler effect. Moving long strings present on the LSS produce velocity fields to the surrounding plasma. Thus, photons scattered for last time on these perturbed last scatterers suffer temperature fluctuations due to the Doppler effect.

The total temperature perturbation may be obtained by superposing the effects of these three mechanisms at all times from $t_{r e c}$ to today. Clearly each 
mechanism involves the superposition of some type of seed present during a given period of time. Therefore, in order to construct the spectrum of the resulting perturbations we must address the following two questions: First, how can we superpose the seeds in order to construct the resulting spectrum and second what is the type of seed corresponding to each mechanism for producing $C M B$ fluctuations?

I will first address the first question. Consider a great circle on the sky (e.g. a meridian) and a seed function $f_{1}^{\Psi}(\theta)$ of angular scale $\Psi$, superposed $N$ times at random positions $\theta_{n}$ on the circle with variable magnitudes $a_{n}$. The resulting pattern will be

$$
f(\theta)=\sum_{n=1}^{N} a_{n} f_{1}^{\Psi}\left(\theta-\theta_{n}\right)=\frac{1}{2 \pi} \sum_{n=1}^{N} a_{n} \sum_{k=-\infty}^{+\infty} \tilde{f}_{1}^{\Psi}(k) e^{i k\left(\theta-\theta_{n}\right)}
$$

where $\tilde{f}_{1}^{\Psi}(k)$ is the Fourier transform of $f_{1}^{\Psi}(\theta)$

$$
\tilde{f}_{1}^{\Psi}(k) \equiv \int_{-\pi}^{+\pi} d \theta f_{1}^{\Psi}(\theta) e^{-i k \theta}
$$

Therefore $\tilde{f}(k)$ (i.e. the Fourier transform of $f(\theta)$ ) can be expressed in terms of $\tilde{f}_{1}^{\Psi}(k)$

$$
\tilde{f}(k)=\tilde{f}_{1}^{\Psi}(k) \sum_{n=1}^{N} a_{n} e^{i k \theta_{n}}
$$

and the corresponding power spectrum $P_{0}(k)$ is easily obtained as the ensemble average of $|\tilde{f}(k)|^{2}$.

$$
P_{0}(k) \equiv<|\tilde{f}(k)|^{2}>=N\left|\tilde{f}_{1}^{\Psi}(k)\right|^{2}<a_{n}^{2}>
$$

The $1 \sigma$ error to $P_{0}(k)$ can also be obtained from the standard deviation of $a_{n}^{2}$. In realistic cases the superposed seed functions $f_{1}^{\Psi}(\theta)$ will be perturbations induced by topological defects which obey a scaling solution and therefore their size is a fixed fraction of the horizon at any given time. This implies that the size of the superposed seeds will be larger at later times due to the expansion of the comoving horizon. Thus when the comoving horizon grows by a factor $\alpha$, the size of the superposed seeds will grow by the same factor while their total number $N$ will be reduced by $\alpha$ since the total number of 
horizons included in the circle will be smaller at that time. By considering $Q$ expansion steps for the comoving horizon we obtain

$$
P_{Q}(k)=\sum_{q=0}^{Q} P_{q}(k) \equiv \sum_{q=0}^{Q} \frac{N}{\alpha^{q}}\left|\tilde{f}_{1}^{\alpha^{q} \Psi}(k)\right|^{2}<a_{n}^{2}>
$$

where $Q$ is determined by the maximum and minimum size of the superposed seeds (or the corresponding horizon scales) as

$$
Q=\frac{\log \left(\frac{\Psi_{\max }}{\Psi_{\min }}\right)}{\log \alpha}
$$

while $N$ is the product of the number of seeds per horizon $M$ times the number of horizon scales included in the circle during the first expansion step when the horizon scale is $\Theta\left(t_{i}\right)$

$$
N\left(t_{i}\right)=M \times \frac{2 \pi}{\Theta\left(t_{i}\right)}
$$

In order to obtain the power spectrum in the case of strings we must define the types of seed functions $f_{1}(\theta)$ that need to be superposed and the initial and final times of superposition for each mechanism generating perturbations.

A long string moving with velocity $v_{s}$ between the LSS and an observer will induce, according to the GKS effect a temperature discontinuity of magnitude 33, 34, 38

$$
\frac{\delta T}{T}=4 \pi G \mu\left(v_{s} \gamma_{s}\right)_{r m s} \hat{k} \cdot(\hat{v} \times \hat{s})
$$

where $\hat{k} \cdot(\hat{v} \times \hat{s})$ is a geometric factor which depends on the relative orientation between the string $\hat{s}$ and the photon unit wavevector $\hat{k}$. Therefore an observer scanning the sky along an arc that itersects the string will detect a temperature step function with amplitude $\frac{\delta T}{T}$ and angular scale $2 \Psi$ depending on the string curvature radius $\xi$ given as a fraction of the angular size $\Theta$ of the horizon at the time the photons interacted with the string $(\Psi(t) \equiv \xi \Theta(t) / 2)$.

Moving long strings present between $t_{e q}$ and $t_{r e c}$ produce velocity perturbations towards the surface they sweep in space. Assuming CDM, these perturbations grow rapidly and form planar density enhancements called wakes. 
Let $\sigma\left(t_{i}, t_{r e c}\right)$ be the surface density of a wake present at $t_{r e c}$ and formed at an earlier time $t_{i}$. The wake, due to its surface density will induce potential fluctuations at a distance $h$ from its surface. The potential fluctuations in turn produce temperature fluctuations on the photons departing from the LSS, with magnitude

$$
\frac{\delta T}{T}=\frac{1}{3} \Phi_{w}\left(x, t_{r e c}\right)=\frac{1}{3} 4 \pi G \sigma\left(t_{i}, t_{r e c}\right)|h(x)|
$$

and size $\Psi$ determined by the scale of coherence of the long string that produced the wake. In $(9) h(x)=x \cos \phi$ is the perpendicular distance to the wake as a function of the angular distance $x$ from the wake on the last scattering surface. The angular distance from the wake over which this perturbation persists is approximatelly $\Psi\left(t_{i}\right)$.Therefore, an observer scanning the sky along an arc that intersects a wake will detect a temperature fluctuation pattern described by equation (9) as a function of the angular scale $x$.

String simulations have shown that the string network contains in addition to long strings, a component of tiny loops with typical sizes about $10^{-4}$ the size of the horizon at any given time. A loop present on the LSS will also induce potential perturbations due both to its energy density and to the dark matter it has accreted. The corresponding temperature fluctuation may be approximated by a function that is 0 on scales much larger than the size of the loop and equal to $\frac{\beta G \mu}{3}\left(\frac{t}{t_{i}}\right)^{2 / 3}$ on smaller scales i.e.

$$
\begin{array}{ll}
\frac{\delta T}{T} & =\frac{1}{3} \Phi_{l}(x, t) \simeq \frac{\beta G \mu}{3}\left(\frac{t}{t_{i}}\right)^{2 / 3} \quad|x| \leq R \\
\frac{\delta T}{T} \simeq 0 \quad|x|>R &
\end{array}
$$

The time dependent factor represents the gravitational growth of the loop induced perturbation on the CDM and $\beta$ is a parameter of order smaller than 10 determining the length of the loop as a function of its radius $R$. Since the typical angular size of a loop present at $t_{r e c}$ is 1 arcsec or less, we expect the effects of loops to be negligible for all present experiments since their resolution is on much larger scales. This is verified from the results shown below.

The velocity perturbations induced by long strings present on the LSS produce velocity fields on the electrons last scatterers of CMB photons. These 
fields induce temperature fluctuations by the Doppler effect. The important perturbations are those produced on the plasma at $t_{r e c}$ since earlier perturbations are damped by photon drag and pressure effects. The magnitude of the produced temperature fluctuations is equal to the projection of the plasma velocity with respect to the observer on the photon unit wavevector

$$
\frac{\delta T}{T}=\hat{k} \cdot \vec{v}=\lambda \pi G \mu v_{s} \gamma_{s} \hat{k} \cdot(\hat{v} \times \hat{s})
$$

where $\lambda$ is defined as

$$
\lambda=\left(1+\frac{\left(1-\frac{T}{\mu}\right)}{2\left(v_{s} \gamma_{s}\right)^{2}}\right)
$$

and is different from 1 in the case of a wiggly string in which the tension $T$ is not equal to the mass per unit length $\mu$. It measures the Newtonian interaction with matter induced by the wiggles of the string. Simulations indicate $\lambda \simeq 6$. The scale of these Doppler perturbations is $\Psi$, determined by the coherence length of long strings present on the LSS.

Having specified the types of seeds that need to be superposed, it is now straightforward to use (5) to obtain the power spectrum component induced by each type of seed. The total spectrum is obtained by summing up the spectrum components.

$$
P(k)=P_{G K S}(k)+P_{W}(k)+P_{L}(k)+P_{D}(k)
$$

A typical spectrum component obtained for the GKS term is

$$
P_{G K S}(k)=\frac{\left.(4 \pi G \mu)^{2}<\left(v_{s} \gamma_{s}\right)^{2}\right)>200 M}{3} \sum_{q=0}^{Q=23} \frac{16 \sin ^{4}\left(\xi 0.008 \alpha^{q} k\right)}{\alpha^{q} k^{2}}
$$

The part $\left.(4 \pi G \mu)^{2}<\left(v_{s} \gamma_{s}\right)^{2}\right)>$ comes from the amplitude of the GKS step function (8), the $1 / 3$ comes by averaging over all string orientations, the $200 M$ is the number of seeds included in the first expansion step occuring at $t_{r e c}$ and the sum is over the Fourier transform of the seed function scaled to expand with the comoving horizon at each expansion step. The assumption that each expansion step occurs when the physical horizon doubles in size implies that $\alpha \simeq 2^{1 / 3} \simeq 1.26$. Decreasing $\alpha$ has the effect of increasing the number of terms $Q$ in the sum (15) but decreasing the value of each term. 
Thus we expect that (15) should not be very sensitive to the precise value of $\alpha$.

The other spectrum components (Wakes, Loops and Doppler) have similar forms expressed in terms of sums 26]. They depend on four parameters: the only free parameter $G \mu$, the parameter $b \equiv M<\left(v_{s} \gamma_{s}\right)^{2}>$, the string coherence length (curvature radius) $\xi$ as a fraction of the horizon scale $\Theta(t)$ $(\Psi(t)=\xi \Theta(t) / 2$ and the parameter $\lambda$ determined by the wiggliness of the string. The three evolution parameters $b, \xi$ and $\lambda$ may be fixed by comparing with numerical simulations while the free parameter $G \mu$ is fixed by comparing with observations (e.g. the COBE detection).

\section{$3 \quad$ Fixing Parameters}

In order to make predictions about ongoing CMB experiments we must determine the only free parameter $G \mu$ as well as the parameters $b, \lambda$ and $\xi$. String simulations [41, 40] indicate that $M \simeq 10$ while $\left(v_{s} \gamma_{s}\right)_{r m s} \simeq 0.15 \mathrm{im}$ plying $b \simeq 0.24$ and $\lambda \simeq 6$. I will verify these values by directly fitting our spectrum with partial CMB spectra obtained by simulations on large angular scales. Bouchet, Bennett and Stebbins 22 (hereafter BBS) have used numerical simulations to calculate the term $P_{G K S}(k)$ for a single expansion step. Their result for the total power on angular scales smaller than $\theta_{*}$ with $t_{i}=t_{r e c}, t_{f} \simeq 2 t_{r e c}$ is

$$
\begin{aligned}
P_{B B S}\left(\theta \leq \theta_{*}, \Theta_{i}=\Theta_{r e c}\right) & =\int_{2 \pi / \theta_{*}}^{\infty} \frac{d^{2} k}{(2 \pi)^{2}} P_{B B S}(k) \\
& =(6 G \mu)^{2}\left(\frac{\theta_{*}^{1.7}}{0.0012+\theta_{*}^{1.7}}\right)^{0.7}
\end{aligned}
$$


The present analysis, focusing on a line across the sky rather than a patch predicts

$$
P_{a n}\left(\theta \leq \theta_{*}, \Theta_{i}=\Theta_{r e c}\right)=2 \int_{2 \pi / \theta_{*}}^{\infty} \frac{d k}{(2 \pi)} P_{K S}^{Q=0}(k)
$$

Figure 1:The total power on scale less than $\theta_{*}$ produced by cosmic strings during one expansion step starting at $t_{r e c}$.

Figure 1 shows $P_{a n}\left(\theta_{*}\right)$ for $b=0.237$ and $\xi=0.45$ (continous line) superimposed on $P_{B B S}\left(\theta_{*}\right)$ (dashed line). It also shows the $1 \sigma$ errors to $P_{a n}\left(\theta_{*}\right)$ obtained from the variance of $a_{n}^{2}$. The values $b=0.237$ and $\xi=0.45$ were chosen in order to obtain the best fit to $P_{B B S}$ but they are in very good agreement with the expected values (obtained for $M \simeq 10,\left(v_{s} \gamma_{s}\right)_{r m s} \simeq 0.15$ and string radius of curvature about half the horizon scale).

Figure 2a shows a superposition of the components of the spectrum (e.g. equation (15) for the GKS term) with the above choice of parameters. The sums were performed using Mathematica[42]. Clearly the GKS term (continous line) dominates on large angular scales $\left(\theta>4^{\circ}\right)$ while the Doppler 
term (long dashed line) is dominant on smaller scales. The contribution of potential perturbations by wakes (dotted line) is less important but is clearly not negligible especially on scales of a few arcmin $(k \simeq 1500)$. Finally, the contibution of loops (short dashed line) is negligible on all scales larger than $2-3 \operatorname{arcmin}(k \leq 8000)$. Figure $2 \mathrm{~b}$ shows the product $k P(k)$ fot the total spectrum (equation (14)) with $1 \sigma$ errors denoted by the dotted lines.

Figure 2a: The four components of the power spectrum of CMB perturbations induced by cosmic strings

One of the most interesting questions that may be addressed using the spectrum of Figure $2 \mathrm{~b}$ is 'What is the effective power spectrum index n, predicted by cosmic strings on COBE angular scales?'. Previous studies 24, 25] have addressed this question without taking into account the effects of potential and Doppler perturbations. The correlation function $C_{1}(\theta)$ for per- 
turbations along a great circle is given in terms of $P(k)$ as

$$
C_{1}(\theta)=<\frac{\delta T}{T}(\phi) \frac{\delta T}{T}(\theta+\phi)>_{\phi}=\frac{1}{(2 \pi)^{2}} \sum_{k=-\infty}^{k=+\infty} P(k) e^{i k \theta}
$$

Figure 2b: The total power spectrum of string perturbations along a great circle on the sky.

For $2 \mathrm{~d}$ maps the corresponding equation is $(l \gg 1, \theta \ll \pi)[35$

$$
C_{2}(\theta) \simeq \frac{1}{(2 \pi)^{2}} \int d^{2} l C_{l} e^{i \vec{l} \cdot \vec{\theta}}
$$

By isotropy we must have $C_{1}(\theta)=C_{2}(\theta)$. It may also be shown that $l^{2} C_{l} \sim$ $l^{n-1}$ where $n$ is the power spectrum index. Since both $k$ and $l$ are Fourier conjugate of $\theta$ we have $k \simeq l$. 
Also (30) and (31) imply (with $\theta \simeq 0$ ) that $P(k) \simeq \pi l C_{l}$ and

$$
k P(k) \sim k^{n-1}
$$

Figure 3a: The best linear fit to the total power spectrum for $5 \leq$ $k \leq 20$.

Figures $3 \mathrm{a}$ and $3 \mathrm{~b}$ show the best linear fit of the $\log$-log plot $k P(k)$ vs $k$, for $5 \leq k \leq 20$ and $5 \leq k \leq 100$ respectively. The best fits give $n=1.35$ (Figure 3a) and $n=1.48$ (Figure $3 \mathrm{~b}$ ). This result indicates that cosmic strings favor values of $n$ somewhat larger than 1 in agreement with recent indications from the Tenerife experiment and the second year data of COBE [43] which favor $n \simeq 1.5$. In contast it is much harder for inflationary models to explain such high values of $n$ 44, 45].

There is a simple analytic way to show that in the sector of the power spectrum where the GKS effect dominates, a scale invariant $(n \simeq 1)$ spectrum should be expected. For $\tilde{f}_{1}^{\Psi}(k) \sim \Psi \tilde{f}_{1}^{\Psi=1}(k \Psi)$ (as in the case of the GKS seed 
functions), (12) may be writen as

$$
P(k)=\sum_{q=0}^{Q} P_{q}(k)=\sum_{q=0}^{Q} \alpha^{q} P_{0}\left(\alpha^{q} k\right) \simeq \alpha P(\alpha k), \quad \Psi_{\max }^{-1} \leq k \leq \Psi_{\min }^{-1}
$$

Figure 3b: The best linear fit to the total power spectrum for $5 \leq$ $k \leq 100$.

Therefore

$$
k P(k) \sim \text { const }
$$

which indicates a scale invariant spectrum for the Kaiser-Stebbins term in the angular scale range $\theta \geq 2^{\circ}\left(k \leq \Psi_{\text {min }}^{-1}\right)$. The Kaiser-Stebbins term plotted in Figure 2a (continous line) is in agreement with this result (the best fit for this component of the spectrum is obtained for $n=1.12$ ). 


\section{Predictions}

The predicted $\frac{\delta T}{T}{ }_{r m s}$ for ongoing experiments can only be obtained after the free parameter $G \mu$ is fixed. This may be achieved by using the COBE detection. Using the DMR filter function $W(k) \simeq e^{-k^{2} / 18^{2}}$ and comparing the observed $\frac{\delta T}{T r m s}=1.1 \pm 0.5$ with the predicted one

$$
{\frac{\delta T}{T_{r m s}}}=(C(0))^{1 / 2}=\left[\frac{1}{2 \pi^{2}} \sum_{k=0}^{\infty} P(k) W(k)\right]^{1 / 2}
$$

the single free parameter $G \mu$ is fixed to the value

$$
G \mu \simeq 1.6 \pm 0.5
$$

Figure 4: The cosmic string predicted correlation function smoothed on COBE scales. Superimposed are the first year COBE data.

Having completely fixed the spectrum we are now in position to use appropriate filter functions to make predictions for ongoing CMB experiments [46] 
on various scales. Figure 4 shows the correlation function obtained by Fourier transforming the power spectrum and smoothed by the COBE filter function. Superimposed are the COBE data 31, 32].

Table 1 shows the approximate location of the maxima $\left(k_{0}\right)$ and the spreads $(\Delta k)$ of the filter functions of several ongoing experiments. It also shows the coresponding detections or upper bounds. The cosmic string predictions were obtained by using (23) with gaussian filter functions of the form

$$
W(k)=e^{-\left(k-k_{0}\right)^{2} / \Delta k^{2}}
$$

and the power spectrum shown in Figure 3. The predictions of the standard model based on inflation $(0.8 \leq n \leq 1.0, \Lambda=0, \mathrm{CDM})$ have been derived in Ref. [4]. From Table 1, it is clear that the predictions of both the standard CDM model and cosmic strings are consistent with detections at the $1 \sigma$ level. However this may well change in the near future as the quality of observations improves.

Table 1:Detections of $\frac{\Delta T}{T}{ }_{r m s} \times 10^{6}$ and the corresponding predictions of the string and inflationary models $(0.8 \leq n \leq 1.0, \Lambda=0)$ normalized on COBE.

\begin{tabular}{|c|c|c|c|c|c|}
\hline Experiment & $k_{0}$ & $\Delta k$ & Detection & Strings & Inflation \\
\hline COBE & 0 & 18 & $11 \pm 2$ & $11 \pm 3$ & $11 \pm 2$ \\
\hline TEN & 20 & 16 & $\leq 17$ & $13 \pm 3$ & $9 \pm 1$ \\
\hline SP91 & 80 & 70 & $11 \pm 5$ & $20 \pm 5$ & $12 \pm 2$ \\
\hline SK & 85 & 60 & $14 \pm 5$ & $19 \pm 4$ & $12 \pm 3$ \\
\hline MAX & 180 & 130 & $\leq 30(\mu P e g)$ & $21 \pm 5.5$ & $16 \pm 5$ \\
\hline MAX & 180 & 130 & $49 \pm 8(G U M)$ & $21 \pm 5.5$ & $16 \pm 5$ \\
\hline MSAM & 300 & 200 & $16 \pm 4$ & $19 \pm 4$ & $24 \pm 6$ \\
\hline OVRO22 & 600 & 350 & - & $13 \pm 4$ & $17 \pm 7$ \\
\hline WD & 550 & 400 & $\leq 12$ & $17.5 \pm 4.5$ & $7 \pm 2$ \\
\hline OVRO & 2000 & 1400 & $\leq 24$ & $13.5 \pm 3.5$ & $7 \pm 3$ \\
\hline
\end{tabular}

In conclusion, I have demonstrated, using a simple analytical method, that the CMB spectrum predicted by the cosmic string model can be calculated in a straightforward way including all the relevant sources of perturbations. I have also shown that the results are consistent with numerical simulations even though their validity extends beyond the resolution of present simulations. Finally I showed that the predicted power spectrum index is 
slightly larger than $1\left(n_{e f f} \simeq 1.4\right)$ and that the predicted rms temperature

fluctuations $\frac{\Delta T}{T}{ }_{r m s}$ are consistent with detections to this date on all angular scales larger than 2-3 arminutes.

This analysis has assumed standard recombination and values of cosmological parameters $\left(\Omega_{0}=1, h=1 / 2, \mathrm{CDM}, \Lambda=0\right)$. It is important to extend these results to less standard cases including reionization or presence of Hot Dark Matter. Work in this direction is in progress.

\section{Acknowledgements}

This work was supported by a CfA Postdoctoral Fellowship.

\section{References}

[1] Guth A. 1981, Phys. Rev. D23, 327.

[2] Guth A. \& Pi S. -Y. 1982, Phys.Rev.Lett.49, 110.

[3] Bardeen J., Steinhardt P. \& M.Turner M. 1983, Phys.Rev. D28, 679.

[4] Bond R., Crittenden R., Davis R., Efstathiou G. \& Steinhardt P. 1994, Phys. Rev. Lett. 72, 13.

[5] Kibble T. W. B. 1976, J.Phys. A9, 1387.

[6] Vilenkin A. 1985, Phys.Rep. 121, 263.

[7] Brandenberger R. 1992, 'Topological Defect Models of Structure Formation After the COBE Discovery of CMB Anisotropies', Brown preprint BROWN-HET-881 (1992), publ. in proc. of the International School of Astrophysics "D.Chalonge", 6-13 Sept.1992, Erice, Italy, ed. N.Sanchez (World Scientific, Singapore, 1993).

[8] Turok N. 1989, Phys. Rev. Lett. 63, 2625.

[9] Turok N. and Brandenberger R. 1986, Phys. Rev. D33, 2175.

[10] Vachaspati T. 1986, Phys. Rev. Lett. 57, 1655.

[11] Stebbins A. et. al. 1987, Ap. J. 322, 1. 
[12] Perivolaropoulos L., Brandenberger R. \& Stebbins A. 1990, Phys.Rev. D41, 1764.

[13] Vachaspati T. \& Vilenkin A. 1991. Phys. Rev. Lett. 67, 1057-1061.

[14] Vollick D. N. 1992, Phys. Rev. D45, 1884.

[15] Hara T. \& Miyoshi S. 1993, Ap. J. 405, 419.

[16] Brandenberger R., Kaiser N., Shellard E. P. S., Turok N. 1987. Phys.Rev. D36, 335.

[17] Albrecht A. \& Stebbins A. 1993. Phys. Rev. Lett. 69, 2615.

[18] Vachaspati T. 1992b, Phys. Rev. D45, 3487.

[19] Vachaspati T. 1992a, Phys.Lett. B282, 305.

[20] Perivolaropoulos L. \& Vachaspati T. 1994, Ap. J. Lett., 423, L77, CfA3590, astro-ph/9303242.

[21] Brandenberger R. \& Turok N. 1986, Phys. Rev. D33, 2182.

[22] Bouchet F. R., Bennett D. P. \& Stebbins A. 1988, Nature 335, 410.

[23] Veeraraghavan S. \& Stebbins A. 1990, Ap.J. 365, 37.

[24] Bennett D., Stebbins A. \& Bouchet F. 1992, Ap.J. Lett. 399, L5.

[25] Perivolaropoulos L. 1993a, Phys.Lett. B298, 305.

[26] Perivolaropoulos L. 1994, Spectral Analysis of CMB Fluctuations Induced by Cosmic Strings, Submitted to the Ap. J., CfA-3591, astroph/9402024.

[27] Gott J. et. al. 1990, Ap.J. 352, 1.

[28] Perivolaropoulos L. 1993b, Phys. Rev. D48, 1530.

[29] Moessner R., Perivolaropoulos L. \& Brandenberger R. 1994, Ap. J., 425 365, astro-ph/9310001. 
[30] Coulson D., Ferreira P., Graham P. \& Turok N. 1993, П in the Sky? CMB Anisotrtopies from Cosmic Defects, PUP-TH-93-1429, hep$\mathrm{ph} / 9310322$.

[31] Smoot G. et. al. 1992, (COBE), Ap. J. Lett. 396, L1.

[32] Wright E. L. et. al. 1992, Ap. J. Lett. 396, L5.

[33] Kaiser N. \& Stebbins A. 1984, Nature 310, 391.

[34] Gott R. 1985, Ap. J. 288, 422.

[35] Efstathiou G. 1989, in 'Physics of the Early Universe', SUSSP 36, 1989, ed. J.Peacock, A.Heavens \& A.Davies (IOP Publ., Bristol, 1990).

[36] Perivolaropoulos L. 1993c, The Fourier Space Statistics of Seed-like Cosmological Perturbations, M.N.R.A.S. in press, CfA-3591, astro$\mathrm{ph} / 9309023$.

[37] Vilenkin A. 1981, Phys.Rev. D23, 852.

[38] Stebbins A. 1988, Ap.J. 327, 584.

[39] Bennett D. \& Bouchet F. 1988, Phys.Rev.Lett. 60, 257.

[40] Allen B. \& Shellard E. P. S. 1990, Phys.Rev.Lett. 64, 119.

[41] Bennett D. \& Bouchet F. 1988, Phys.Rev.Lett. 60, 257.

[42] Wolfram S. 1991, Mathematica version 2.0, Addison-Wesley.

[43] Bennett C. L. et. al. 1994, Cosmic Temperature Fluctuations from Two Years of COBE DMR Observations, submitted to Ap. J., (ASTROPH9401012).

[44] Lyth D. \& Liddle A. 1994,Observational Constraints on the Spectral Index, Contribution to the 1993 Capri CMB Workshop. SUSSEX-AST 93/12-1, astro-ph/9401014.

[45] Steinhardt P. 1993, private communication. 
[46] TEN: Watson R. A. et. al. 1992, Nature 357, 660.

SP91: Gaier T. et.al. 1992, (SP91), Ap. J. Lett. 398, L1.

SP91: Schuster J. et. al. 1993, (SP91), Ap. J. Lett. 412, L47.

MSAM: Cheng E. S. et. al. 1993,A Measurement of the Medium Scale

Anisotropy in the CMB, preprint MSAM-93A.

SK: Wollack E. J. et. al. 1993, Ap. J. Lett. 419, L49.

MAX1: Meinhold P. et. al. 1993, Ap. J. Lett. 409, L1.

MAX2: Gunderson J. et. al. 1993, Ap. J. Lett. 413, L1.

WD: Tucker G. S. et. al. 1993, (WD), Princeton preprint.

OVRO: Readhead A.C.S. et.al. 1989, (OVRO), Ap. J. 346, 556. 